\title{
ARTICLE OPEN Ketogenic diets inhibit mitochondrial biogenesis and induce cardiac fibrosis
}

\author{
Sha $\mathrm{Xu}^{1,2}$, Hui Tao ${ }^{3}$, Wei $\mathrm{Cao}^{3}$, Li Cao $\mathbb{D}^{1}$, Yan Lin ${ }^{1}$, Shi-Min Zhao ${ }^{1,2}$, Wei $\mathrm{Xu}^{1,2}$, Jing Cao ${ }^{4}$ and Jian-Yuan Zhao $\mathbb{D}^{1,2,4}$
}

In addition to their use in relieving the symptoms of various diseases, ketogenic diets (KDs) have also been adopted by healthy individuals to prevent being overweight. Herein, we reported that prolonged KD exposure induced cardiac fibrosis. In rats, KD or frequent deep fasting decreased mitochondrial biogenesis, reduced cell respiration, and increased cardiomyocyte apoptosis and cardiac fibrosis. Mechanistically, increased levels of the ketone body $\beta$-hydroxybutyrate ( $\beta-\mathrm{OHB})$, an HDAC2 inhibitor, promoted histone acetylation of the Sirt7 promoter and activated Sirt7 transcription. This in turn inhibited the transcription of mitochondrial ribosome-encoding genes and mitochondrial biogenesis, leading to cardiomyocyte apoptosis and cardiac fibrosis. Exogenous $\beta-O H B$ administration mimicked the effects of a KD in rats. Notably, increased $\beta-O H B$ levels and SIRT7 expression, decreased mitochondrial biogenesis, and increased cardiac fibrosis were detected in human atrial fibrillation heart tissues. Our results highlighted the unknown detrimental effects of KDs and provided insights into strategies for preventing cardiac fibrosis in patients for whom KDs are medically necessary.

\section{INTRODUCTION}

The low-carbohydrate, high-fat ketogenic diet (KD) is a remarkably effective treatment for medically intractable epilepsy and has been applied in the clinical setting for over 70 years. ${ }^{1}$ In addition, KDs have been widely applied in the clinical treatment of various diseases, such as diabetes, ${ }^{2}$ cancer, ${ }^{3,4}$ and neurological disorders, including Alzheimer's disease and Parkinson's disease. ${ }^{5} \mathrm{KDs}$ are also used by healthy individuals, predominantly to promote weight loss. $^{6}$

Consumption of a KD forces the body to use fats rather than carbohydrates to generate energy. Three major forms of ketone bodies, namely acetoacetate (AcAc), $\beta$-hydroxybutyrate ( $\beta-\mathrm{OHB})$, and acetone, are generated in the liver during fatty acid oxidation and transported to extrahepatic tissues by the circulatory system. Circulating total ketone body concentrations in healthy adult humans normally exhibit circadian oscillations of $\sim 100-250 \mu \mathrm{M}$. However, levels can reach 1-8 mM after KD consumption, prolonged exercise, or deep fasting and can be as high as $25 \mathrm{mM}$ under pathological conditions, such as diabetic ketoacidosis. ${ }^{7-10} \beta-\mathrm{OHB}$ accounts for $70 \%$ of ketone bodies and has been suggested to be beneficial because it not only serves as a vital alternative metabolic fuel source in the fed, fasted, and starved states ${ }^{11}$, but also exerts antioxidative, ${ }^{12}$ antiaging, ${ }^{13}$ and antiinflammatory effects. ${ }^{14}$

Although numerous reports have acknowledged the beneficial effects of $\beta-\mathrm{OHB}$, its safety has been challenged by certain clinical lines of evidence related to its effects on cardiovascular health. For example, the concentration of $\beta-\mathrm{OHB}$ in heart tissues is significantly higher in patients with atrial fibrillation (AF). ${ }^{15}$ In addition, increased circulating $\beta-\mathrm{OHB}$ is independently associated with major adverse cardiovascular events in patients undergoing hemodialysis. ${ }^{16}$ Moreover, diabetes, which is usually associated with high levels of ketone bodies, constitutes an independent risk factor for cardiovascular diseases, including $A F$, coronary heart disease, and stroke. ${ }^{17,18}$ The potential detrimental effects of $\beta-O H B$ have also been implicated in clinical studies, in which KD has been practiced. For example, the occurrence of cardiovascular disease of unknown etiology has frequently been described in those in the KD group in various studies. ${ }^{19-21}$ Moreover, a 25 -year follow-up study of a large cohort found that a low-carbohydrate diet was associated with increased mortality, ${ }^{22}$ although it was not clear whether the KD directly increased the incidence of cardiovascular disease, a major factor that affects life expectancy. ${ }^{23}$ Taken together, these findings suggest that KD consumption or $\beta-O H B$ accumulation may increase the risks of cardiovascular disease, suggesting that long-term consumption of a KD should be carefully considered.

In this study, we examined the effects of a KD and $\beta-O H B$ accumulation on cardiovascular biology and health in cultured cells, animal models, and clinical samples in order to shed light on the potential negative effects of $\mathrm{KDs}$ and to elucidate the underlying mechanisms.

\footnotetext{
'Zhongshan Hospital of Fudan University, Obstetrics \& Gynecology Hospital of Fudan University, State Key Lab of Genetic Engineering, School of Life Sciences, Key Laboratory of Reproduction Regulation of NPFPC, and Institutes of Biomedical Sciences, Fudan University, 200438 Shanghai, China; ${ }^{2}$ Collaborative Innovation Center for Biotherapy, West China Hospital, Sichuan University, 610041 Chengdu, China; ${ }^{3}$ Department of Cardiothoracic Surgery, Second Hospital of Anhui Medical University, and Cardiovascular Research Center, Anhui Medical University, 230601 Hefei, China and ${ }^{4}$ Department of Anatomy and Neuroscience Research Institute, School of Basic Medical Sciences, Zhengzhou University, 450001 Zhengzhou, China

Correspondence: Jian-Yuan Zhao (zhaojy@fudan.edu.cn)

These authors contributed equally: Sha Xu, Hui Tao, Wei Cao.
}

Received: 7 May 2020 Revised: 31 August 2020 Accepted: 3 November 2020

Published online: 09 February 2021 
Table 1. Echocardiographic data from rats fed a normal diet, ketogenic diet, or caloric restriction diet

\begin{tabular}{|c|c|c|c|}
\hline & Normal diet & $\begin{array}{l}\text { Caloric } \\
\text { restriction }\end{array}$ & Ketogenic diet \\
\hline $\begin{array}{l}\text { Heart rate, } \\
\text { beats } / \mathrm{min}\end{array}$ & $321.60 \pm 11.06$ & $334.60 \pm 16.65$ & $363.20 \pm 8.47^{*}$ \\
\hline $\mathrm{LAD}, \mathrm{mm}$ & $3.93 \pm 0.17$ & $3.87 \pm 0.19$ & $4.56 \pm 0.23 * *$ \\
\hline $\mathrm{RAD}, \mathrm{mm}$ & $4.08 \pm 0.18$ & $4.01 \pm 0.20$ & $4.24 \pm 0.20$ \\
\hline LVAWd, mm & $1.89 \pm 0.06$ & $1.91 \pm 0.05$ & $1.96 \pm 0.12$ \\
\hline LVPWd, mm & $1.79 \pm 0.06$ & $1.76 \pm 0.09$ & $2.08 \pm 0.11 *$ \\
\hline LVDd, mm & $7.46 \pm 0.06$ & $7.54 \pm 0.11$ & $7.83 \pm 0.14^{*}$ \\
\hline LVDs, mm & $5.81 \pm 0.12$ & $5.96 \pm 0.10$ & $6.64 \pm 0.31 * *$ \\
\hline LVFS, \% & $42.89 \pm 2.99$ & $43.99 \pm 2.79$ & $40.14 \pm 4.55$ \\
\hline LV mass, mg & $997.60 \pm 26.37$ & $988.28 \pm 19.97$ & $1021.06 \pm 22.12$ \\
\hline RVAW, mm & $0.54 \pm 0.03$ & $0.55 \pm 0.04$ & $0.63 \pm 0.04 * *$ \\
\hline $\mathrm{RVD}, \mathrm{mm}$ & $3.80 \pm 0.07$ & $3.82 \pm 0.08$ & $3.87 \pm 0.14$ \\
\hline LPV (S), cm/s & $52.22 \pm 1.42$ & $51.88 \pm 1.52$ & $52.32 \pm 2.46$ \\
\hline LPV (D), cm/s & $35.24 \pm 1.02$ & $35.48 \pm 1.23$ & $35.64 \pm 2.51$ \\
\hline \multicolumn{4}{|c|}{$\begin{array}{l}\text { Values are mean } \pm \text { SEM }(n=6) \\
L A D \text { left atrial diameter, } R A D \text { right atrial diameter, } L V A W d \text { left ventricular } \\
\text { anterior wall thickness, } L V P W d \text { left ventricular posterior wall thickness, } L V D d \\
\text { left ventricular dimension in diastole, } L V D \text { s left ventricular dimension in } \\
\text { systole, } L V F S \text { left ventricular fractional shortening, } R V A W \text { right ventricular } \\
\text { anterior wall thickness, RVD right ventricular dimension, } L P V \text { left pulmonary } \\
\text { venous flow, } S \text { Systolic, } D \text { diastolic } \\
{ }^{*} p<0.05, *{ }^{*} p<0.01 \text { versus the normal diet-fed group according to } \\
\text { Student's } t \text { tests }\end{array}$} \\
\hline
\end{tabular}

\section{RESULTS}

Long-term KD exposure resulted in cardiac fibrosis

To survey the potential pathological effects of a KD on cardiac disease, we fed rats either a KD or normal diet and monitored changes in the rat heart (Supplementary Fig. 1a-c). After 16 weeks, besides a decrease in body weight (Supplementary Fig. 1d), fat mass (Supplementary Fig. 1e), and blood pressure (Supplementary Fig. 1f, g) in KD-fed rats, we observed increased heart rates and impaired cardiac function, as evaluated by echocardiography (Table 1). In KD-fed rats, the left ventricular posterior wall thickness (LVPWd) increased significantly compared with normal diet-fed rats, indicating a compensatory increase in cardiomyocytes, used to enhance cardiac contractility (Table 1). If the left heart function was decompensated, the blood accumulated in the pulmonary circulation. The right ventricular anterior wall thickness (RVAW) also increased, indicating increased pulmonary circulation resistance and corresponding thickening of the right ventricular myocardium. Furthermore, the left atrial diameter (LAD), left ventricular dimension in diastole (LVDd), and left ventricular dimension in systole (LVDs) increased in KD-fed rats, indicating that cardiac function was decompensated and entered a state of failure (Table 1). Taken together, the echocardiography results revealed impaired cardiac function in KD-fed rats.

Considering the fact that increased cardiac fibrosis is associated with cardiac hypertrophy, dilatation, ${ }^{24-26}$ and the onset of $\mathrm{AF}^{27}$ and that significantly increased ketone bodies were observed in the cardiac tissues of patients with $\mathrm{AF}^{15}$ we next examined the cardiac fibrosis levels in rat atrial tissues. In accordance with impaired cardiac function, we observed the occurrence of fibrosis in the atrial tissues of KD-fed but not normal diet-fed rats, as analyzed by atrial tissue staining (Fig. 1a and Supplementary Fig. 2a). We also detected the expression levels of protein markers of fibrosis using both immunohistochemistry (IHC) and western blotting. Indeed, type I collagen, type III collagen, and a-smooth muscle actin (a-SMA) levels were increased in atrial tissues from
KD-fed rats (Fig. 1b, c and Supplementary Fig. 2b, c). These results indicated that long-term KD feeding may cause cardiac fibrosis.

Because a KD usually provides fewer calories than a carbohydrate-rich diet, we employed caloric restriction (CR) in another group of rats as a control to determine whether KD induced cardiac fibrosis was caused by an insufficient supply of energy. We found CR did not induce cardiac fibrosis and cardiac function impairment in rats (Fig. 1a-c, Supplementary Figs. 1 and 2 , and Table 1). Moreover, the KD caused increased levels of $\beta-\mathrm{OHB}$ and AcAc, the predominant forms of ketone bodies, in both plasma and heart tissues (Fig. 1d, e). In contrast, CR only induced a mild elevation of $\beta-\mathrm{OHB}$ and AcAc (Fig. 1d, e). These observations suggested that a considerable elevation in ketone bodies may account for the onset of cardiac fibrosis in rats.

\section{Increased $\beta-\mathrm{OHB}$ resulted in cardiac fibrosis}

To further elucidate whether and which ketone bodies induced fibrosis, we increased the levels of either $\beta-\mathrm{OHB}$ or AcAc in rats by intraperitoneal injection (Supplementary Fig. 3a-d) because $\beta-\mathrm{OHB}$ and AcAc were the predominant forms of ketone bodies elevated after KD feeding (Fig. 1d, e). During the 16-week injection period, water intake, daily calorie intake, body weight, fat mass, and blood pressure were not affected by either $\beta-O H B$ or AcAc injection (Supplementary Fig. 3e-j). We found that increased levels of $\beta-O H B$, but not AcAc, resulted in impaired cardiac function, as evidenced by increased LAD, LVDs, LVDd, LVPWd, and RVAW in echocardiography analysis (Table 2). Furthermore, we observed the occurrence of cardiac fibrosis in $\beta-\mathrm{OHB}$ intraperitoneal injected rats, as evident by atrial staining (Fig. If and Supplementary Fig. 4a) and analysis of cardiac fibrosis molecular markers, namely type I collagen, type III collagen, and a-SMA (Fig. 1g, $h$ and Supplementary Fig. $4 b, c)$. We also observed that frequent deep fasting (Supplementary Fig. 5a, b) induced high levels of $\beta-\mathrm{OHB}$ (Supplementary Fig. $5 \mathrm{c}$ ) and molecular phenotypes of cardiac fibrosis, including characteristic fiber staining results (Supplementary Fig. 5d), and expression of collagen and a-SMA (Supplementary Fig. 5e). Together, these findings supported the theory that increased levels of $\beta-\mathrm{OHB}$ resulted in cardiac fibrosis.

\section{$\beta$-OHB promoted cardiomyocyte apoptosis}

We next investigated how $\beta-\mathrm{OHB}$ induced cardiac fibrosis. In cultured human cardiomyocytes (HCM), rat cardiomyoblasts $(\mathrm{H} 9 \mathrm{C} 2)$, and mouse cardiac muscle cells $(\mathrm{HL}-1)$, increased levels of $\beta$-OHB led to elevated rates of apoptosis; however, $\beta-O H B$ did not induce significant increases in apoptosis in fibroblasts, such as mouse embryonic fibroblasts (MEFs) and 3T3 cells (Fig. 2a). In primary cells isolated from neonatal mice, $\beta-\mathrm{OHB}$ treatment also led to an increase in apoptosis in primary mouse cardiomyocytes, but not in primary mouse cardiac fibroblast (Fig. 2b). In accordance with increased apoptosis, elevated $\beta-O H B$ led to increases in the levels of cleaved caspase 3 in HCM and $\mathrm{H} 9 \mathrm{C} 2$ cells, but not in MEFs (Fig. 2c). Moreover, in rat atrial tissue, we confirmed that high $\beta$-OHB levels, induced by either KD feeding or $\beta-O H B$ injection, led to increased rates of apoptosis, as evidenced by in situ TUNEL assays (Fig. 2d) and detection of the levels of cleaved caspase 3 in rat atrial tissue (Fig. 2e). These results suggested that a high concentration of $\beta$-OHB caused cell-typespecific cardiomyocyte apoptosis and may underlie $\beta-\mathrm{OHB}$ induced cardiac fibrosis, which is known to be caused by cardiomyocyte apoptosis. $^{28,29}$

We next evaluated the mechanisms underlying cardiomyocyte apoptosis induced by $\beta-\mathrm{OHB}$. First, given that $\beta-\mathrm{OHB}$ constitutes an important alternative energy source that can be catalyzed into acetyl-CoA for processing through the citrate cycle, ${ }^{30}$ we examined whether $\beta$-OHB affected energy metabolism through enhancing the ketolysis pathway. By measuring the expression of proteins within the ketolysis pathway in $\mathrm{H} 9 \mathrm{C} 2, \mathrm{HL}-1$, and $\mathrm{HCM}$ cells treated with or without $\beta-\mathrm{OHB}$, we observed decreased expression 


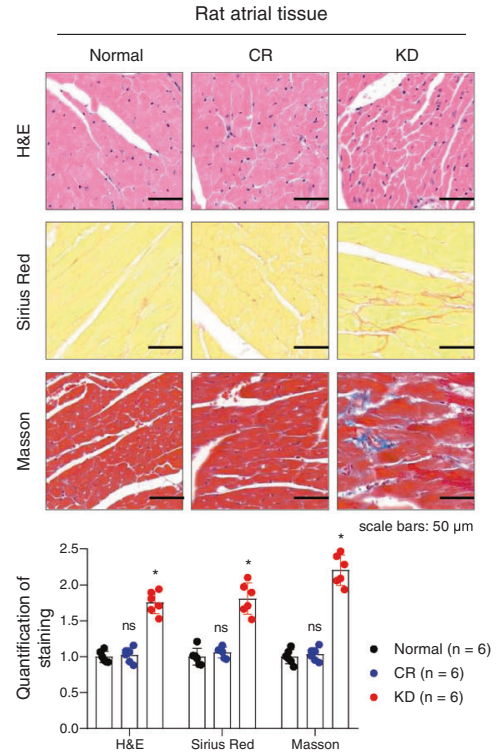

d
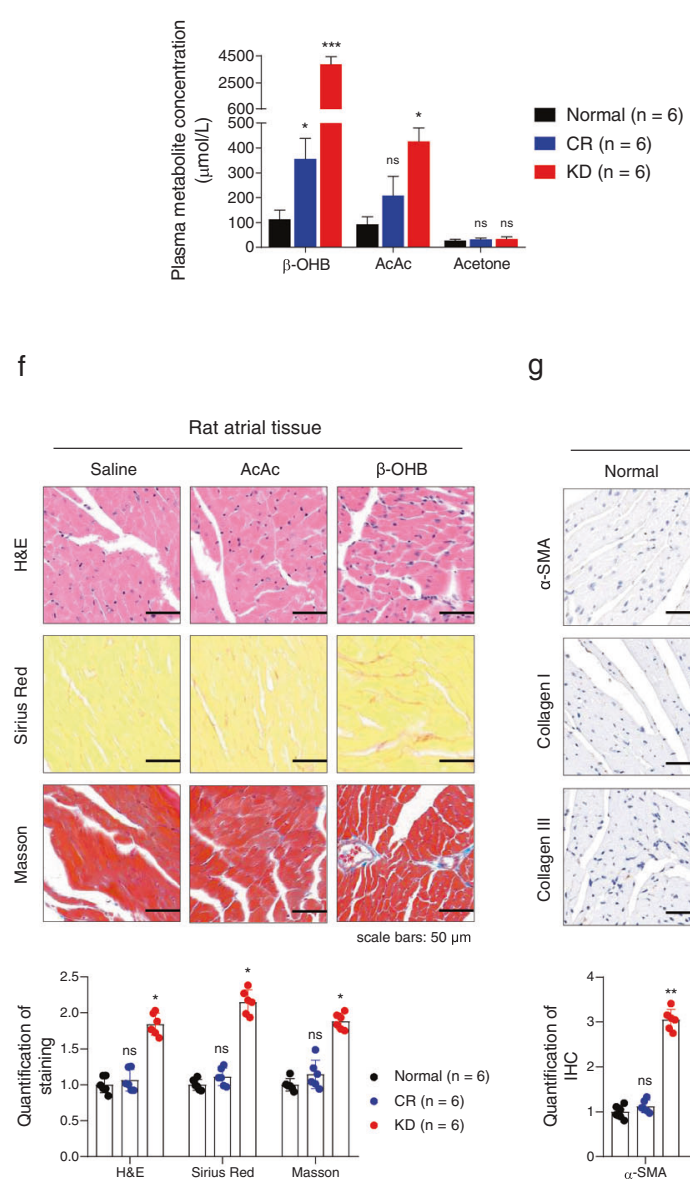

g

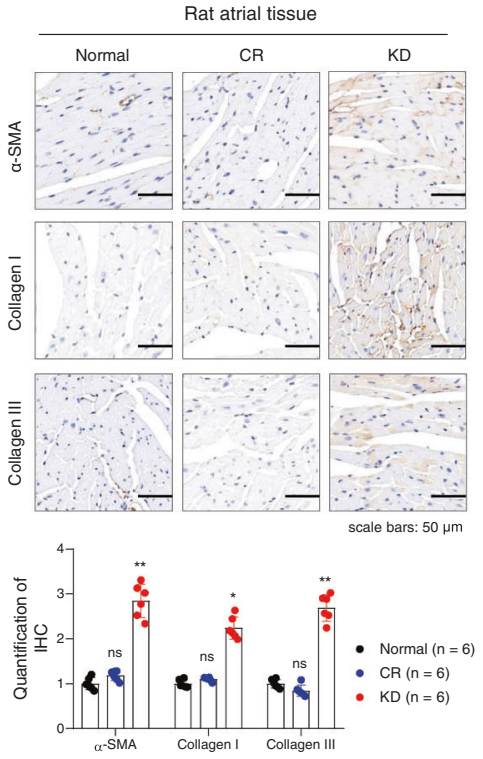

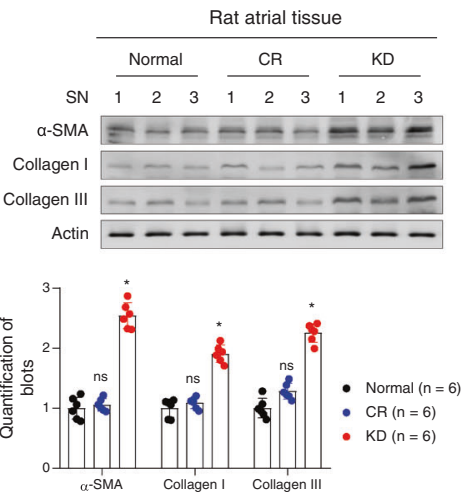

e

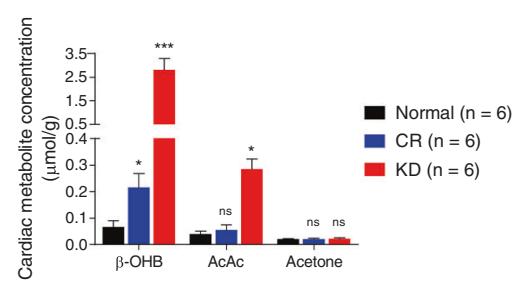

$\mathrm{h}$
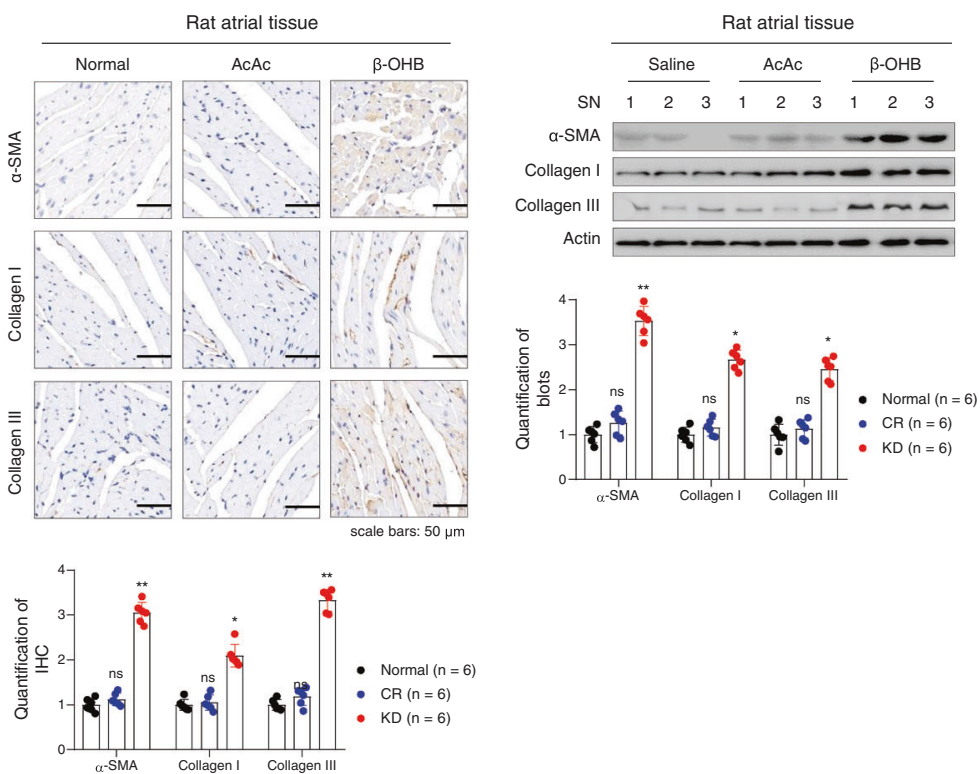

of $\beta$-hydroxybutyrate dehydrogenase 1 (BDH1) and succinylCoA:3-ketoacid CoA transferase (SCOT) in $\beta$-OHB-treated cells (Fig. 2f), and atrial tissues from KD-fed and $\beta$-OHB-injected rats (Fig. 2g), suggesting that increased input of ketone bodies did not enhance ketolysis. We next evaluated the glycolytic and citrate metabolic flux in cultured cell lines and primary cardiomyocytes. Decreased levels of citrate cycle metabolites and increased levels of the glycolytic metabolites pyruvate and lactate were observed in $\beta$-OHB-treated cells (Fig. $2 \mathrm{~h}$ ). Together with our results showing that decreased levels of citrate cycle metabolites and increased 
Fig. 1 Increased $\beta$-OHB induced cardiac fibrosis in animal models. a, b H\&E, Sirius Red, and Masson trichrome staining (a) results in atrial tissues from rats fed a normal diet, KD, or CR diet, and immunohistochemical (IHC) analysis of $\alpha$-SMA, collagen I, and collagen III in atrial tissues from rats according to diet (b). Representative results (top) and summary of quantitative analysis are shown. c Western blotting analysis of $\alpha$-SMA, collagen I, and collagen III in atrial tissues of rats fed the different diets. $\mathbf{d}$, e Ketone body concentrations in the plasma (d) and atrial tissues (e) of rats fed the different diets. $\mathbf{f}, \mathbf{g ~ H} \& \mathrm{E}$, Sirius Red, and Masson staining results of atrial tissues from rats intraperitoneally injected with saline, ACAc, or $\beta-\mathrm{OHB}(\mathbf{f})$ and IHC analysis of $\alpha-\mathrm{SMA}$, collagen I, and collagen III in atrial tissues from rats according to treatment (g). Representative results (top) and summary of quantitative analysis are shown. $\mathbf{h}$ Western blotting analysis of $\alpha$-SMA, collagen I, and collagen III in atrial tissues of rats according to treatment. Data shown are means \pm standard errors. ${ }^{\text {ns }} p>0.05,{ }^{*} p<0.05,{ }^{* *} p<0.01,{ }^{* * *} p<0.001$ versus the corresponding control group according to Student's $t$ tests. Some full-length blots are presented in the Supplementary Fig. 13

\begin{tabular}{|c|c|c|c|}
\hline & Saline & $A C A C$ & $\beta-\mathrm{OHB}$ \\
\hline $\begin{array}{l}\text { Heart rate, } \\
\text { beats/min }\end{array}$ & $318.80 \pm 8.53$ & $324.00 \pm 13.75$ & $354.80 \pm 15.69 *$ \\
\hline LAD, mm & $3.84 \pm 0.23$ & $3.96 \pm 0.23$ & $4.50 \pm 0.22^{* *}$ \\
\hline $\mathrm{RAD}, \mathrm{mm}$ & $4.10 \pm 0.18$ & $4.11 \pm 0.19$ & $4.19 \pm 0.21$ \\
\hline LVAWd, mm & $1.91 \pm 0.05$ & $1.92 \pm 0.08$ & $2.02 \pm 0.12$ \\
\hline LVPWd, mm & $1.81 \pm 0.05$ & $1.79 \pm 0.04$ & $2.16 \pm 0.24^{*}$ \\
\hline LVDd, mm & $7.47 \pm 0.06$ & $7.58 \pm 0.14$ & $7.73 \pm 0.18^{*}$ \\
\hline LVDs, mm & $5.78 \pm 0.08$ & $5.87 \pm 0.17$ & $6.57 \pm 0.53^{*}$ \\
\hline LVFS, \% & $42.69 \pm 2.08$ & $44.36 \pm 2.52$ & $40.40 \pm 4.11$ \\
\hline LV mass, mg & $1000.20 \pm 18.10$ & $1003.60 \pm 24.22$ & $1026.00 \pm 23.91$ \\
\hline RVAW, mm & $0.53 \pm 0.03$ & $0.56 \pm 0.04$ & $0.63 \pm 0.03 * *$ \\
\hline RVD, mm & $3.82 \pm 0.10$ & $3.77 \pm 0.08$ & $3.78 \pm 0.07$ \\
\hline LPV (S), cm/s & $52.04 \pm 1.25$ & $52.14 \pm 1.67$ & $51.62 \pm 3.33$ \\
\hline LPV (D), cm/s & $36.42 \pm 1.16$ & $35.72 \pm 1.46$ & $35.44 \pm 2.24$ \\
\hline \multicolumn{4}{|c|}{$\begin{array}{l}\text { Values are mean } \pm \text { SEM }(n=6) \\
L A D \text { left atrial diameter, RAD right atrial diameter, } L V A W d \text { left ventricular } \\
\text { anterior wall thickness, } L V P W d \text { left ventricular posterior wall thickness, } L V D d \\
\text { left ventricular dimension in diastole, } L V D \text { s left ventricular dimension in } \\
\text { systole, } L V F S \text { left ventricular fractional shortening, RVAW right ventricular } \\
\text { anterior wall thickness, RVD right ventricular dimension, } L P V \text { left pulmonary } \\
\text { venous flow, S Systolic, } D \text { diastolic } \\
{ }^{*} p<0.05,{ }^{* *} p<0.01 \text { versus the saline group according to Student's } t \text { tests }\end{array}$} \\
\hline
\end{tabular}

levels of the glycolytic metabolites were also observed in atrial tissues from rats with high $\beta$-OHB levels (Fig. 2i), these findings indicated that high $\beta-\mathrm{OHB}$ did not enhance mitochondrial metabolism. Instead, increased $\beta-\mathrm{OHB}$ may lead to reduced mitochondrion numbers because the levels of some mitochondrial enzymes decreased (Fig. 2f, g).

\section{$\beta-\mathrm{OHB}$ decreased mitochondrion levels in cardiomyocyte}

Next, we evaluated the number of mitochondria in response to $\beta-\mathrm{OHB}$ treatment. Our results showed that $\beta-\mathrm{OHB}$ treatment decreased MitoTracker Green staining levels (Fig. 3a) and decreased the ratio of mitochondrial (mt) DNA to nucleic DNA (Fig. 3b) in $\mathrm{HCM}, \mathrm{H} 9 \mathrm{C2}$, and $\mathrm{HL}-1$ cells, in a concentrationdependent manner. In primary mouse cardiomyocytes isolated from neonatal mice, $\beta-\mathrm{OHB}$ treatment also led to a decrease in mitochondrion mass, as determined by measuring the ratio of mtDNA to nucleic DNA (Fig. 3c). In accordance with the observations in cultured cells, in rats fed a KD or injected intraperitoneally with $\beta-\mathrm{OHB}$, significant decreases in cardiac mitochondrion mass were observed compared with those in untreated animals, as determined by measuring the ratio of mtDNA to nucleic DNA (Fig. 3d, e). Moreover, the mtDNA damage levels were not altered significantly in $\beta$-OHB-treated cells (Supplementary Fig. 6a) and cardiomyocytes from rats fed a KD or injected intraperitoneally with $\beta-\mathrm{OHB}$ (Supplementary Fig. $6 \mathrm{~b}, \mathrm{c}$ ).
These results indicated that $\beta-\mathrm{OHB}$ decreased mitochondrial numbers in cultured cells and rat heart tissue.

Consistent with this observation, mitochondrial respiration was significantly reduced after $\beta-\mathrm{OHB}$ treatment in both $\mathrm{H} 9 \mathrm{C} 2$ and HL-1 cells (Fig. 3f, g). We also observed decreased mitochondrial respiration in primary cardiomyocytes isolated from KD-fed or $\beta$-OHB-injected rats (Fig. 3h, i). Furthermore, oxyrase-induced hypoxia caused apoptosis, and saturated the proapoptotic effects of $\beta-\mathrm{OHB}$ in $\mathrm{H} 9 \mathrm{C} 2$ and HL-1 cells (Fig. $3 \mathrm{j}$ ) and primary mouse cardiomyocytes (Fig. $3 \mathrm{k}$ ). These results were consistent with the hypothesis that a decrease in mitochondrion numbers may lead to cardiomyocyte apoptosis, which has been shown to be induced by hypoxia. ${ }^{31-35}$ In addition, we observed that $\beta$-OHB slightly decreased the mitochondrial mass in MEFs and primary mouse cardiac fibroblasts (Supplementary Fig. 7a, b), and did not exhibit strong inhibitory effects on mitochondrial respiration (Supplementary Fig. 7c, d). Accordingly, the apoptosis levels of MEFs and primary cardiac fibroblasts were not altered in response to hypoxia and $\beta-\mathrm{OHB}$ treatments (Supplementary Fig. $7 e$, f). These observations suggested that $\beta$-OHB caused celltype-specific cardiomyocyte apoptosis through inhibiting mitochondrial respiration, consistent with previous findings demonstrating that cardiomyocytes are more sensitive to mitochondrial inhibition than fibroblasts. ${ }^{36-38}$ Taken together, these results supported the hypothesis that high levels of $\beta-\mathrm{OHB}$ may induce cardiomyocyte apoptosis by inhibiting mitochondria-mediated oxygen utilization.

$\beta$-OHB impaired mitochondrial biogenesis by activating SIRT7 By monitoring protein markers of mitochondrial biogenesis and degradation, we found that $\beta-\mathrm{OHB}$ decreased protein levels of mitochondrial ribosomal protein L16 (MRPL16), mitochondrial ribosomal protein L24 (MRPL24), and G elongation factor mitochondrial 2 (GFM2), markers of mitochondrial biogenesis (Fig. 4a). ${ }^{39} \beta-O H B$ also decreased GFM2, MRPL16, and MRPL24 mRNA levels (Fig. 4b). Moreover, the expression of PTEN-induced kinase 1 (PINK1) and parkin (PRKN), along with the relative ratio of LC3-I to LC3-II, markers of mitophagy, were not affected by $\beta-O H B$ treatment, suggesting that $\beta-\mathrm{OHB}$ did not promote mitochondrial autophagy (Fig. 4a). These results collectively suggested that $\beta-\mathrm{OHB}$ inhibited cell respiration via the suppression of mitochondrial biogenesis.

In an attempt to identify genes that were transcriptionally regulated by $\beta-\mathrm{OHB}$, we evaluated the gene expression profiles of $\mathrm{H} 9 \mathrm{C} 2$ cells with or without $\beta-\mathrm{OHB}$ treatment. The results showed that the transcription of Sirt4 and Sirt7 was activated by $\beta-\mathrm{OHB}$ (Supplementary Fig. 8a). Because other markers of mitochondrial biogenesis, such as Polrmt, Tfb1m, and Tfam, were not reduced after $\beta-\mathrm{OHB}$ treatment and because SIRT7 specifically regulates MRPL16, MRPL24, and GFM2, ${ }^{40}$ we hypothesized that $\beta-O H B$ blocked mitochondrial activity via SIRT7-mediated transcriptional regulation. In cultured $\mathrm{H} 9 \mathrm{C} 2, \mathrm{HL}-1$, and $\mathrm{HCM}$ cells, we confirmed that $\beta$-OHB increased SIRT7 expression at both the mRNA and protein levels in a concentration-dependent manner (Fig. 4c, d). Knockdown of SIRT7 increased the transcription of Gfm2 and Mrpl24, and attenuated the inhibitory effects of $\beta$-OHB treatment on Gfm2 and Mrpl24 transcription (Fig. 4e). Conversely, 
a

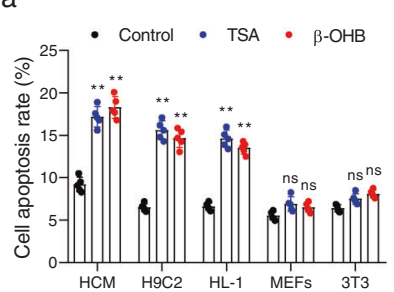

b

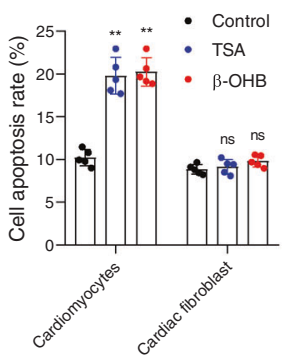

d

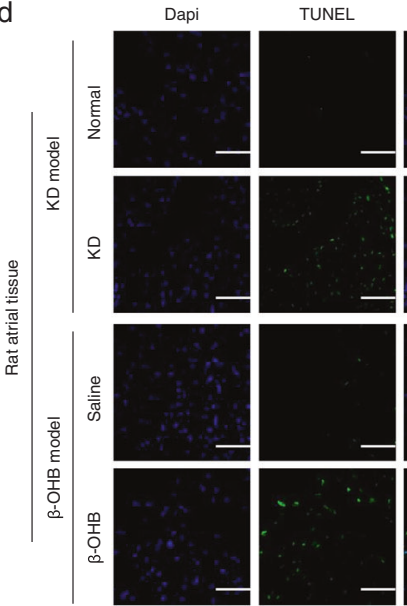

Merged
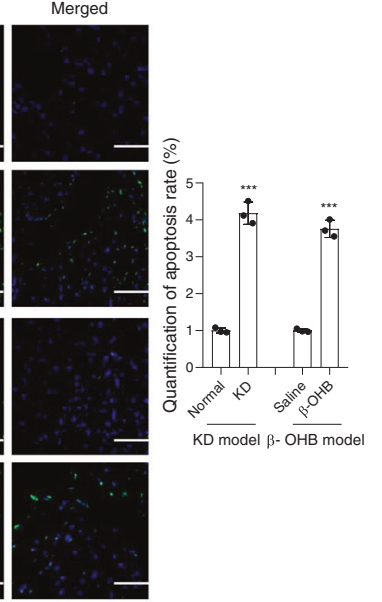

g
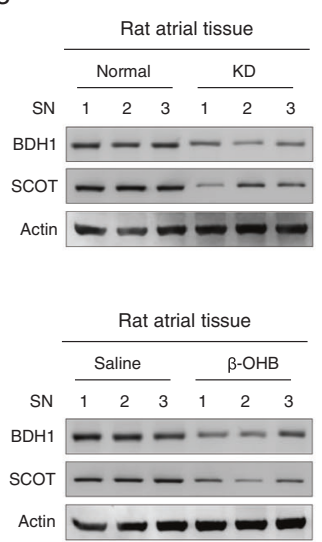

$\mathrm{h}$
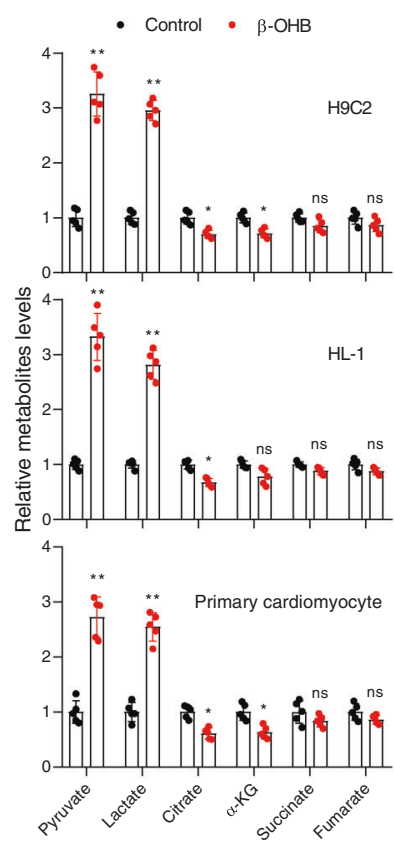
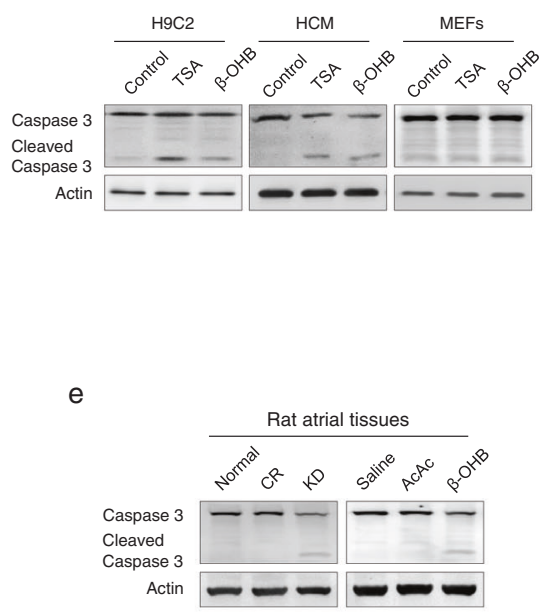

f

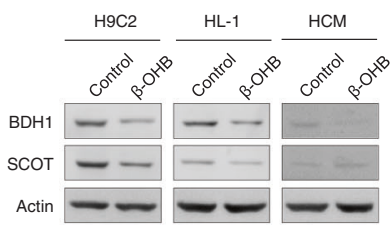

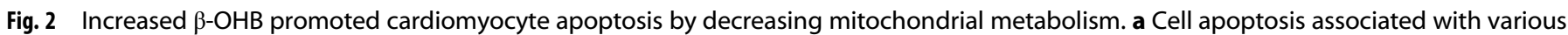

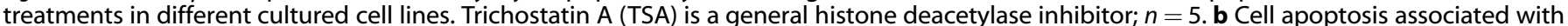
various treatments in primary cells isolated from neonatal mice; $n=5$. c Western blotting analysis of caspase 3 in different cell lines with various treatments. d TUNEL analysis of atrial tissues from rats with different treatments; $n=3$. e Western blotting analysis of caspase 3 in atrial tissues from rats with various treatments. $\mathbf{f}$ Western blotting analysis of BDH1 and SCOT in different cell lines treated with or without $\beta$-OHB. g Western blotting analysis of BDH1 and SCOT in atrial tissues from rats with various treatments. $\mathbf{h}$ Metabolite concentrations in cell lines and

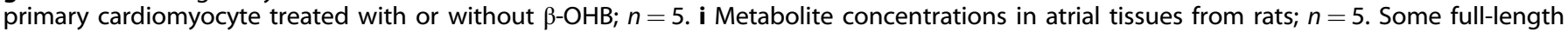
blots are presented in the Supplementary Fig. 13 

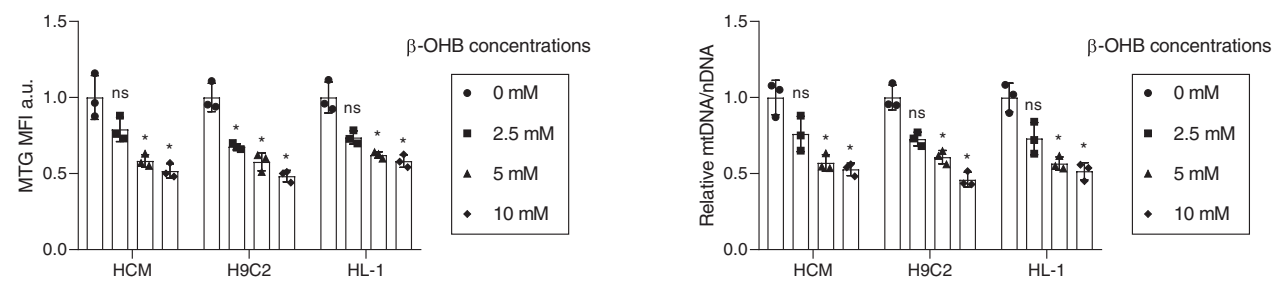

C

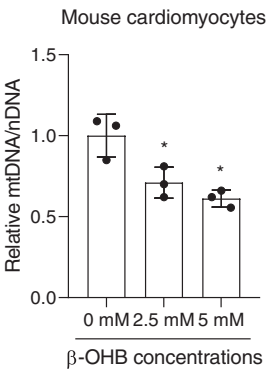

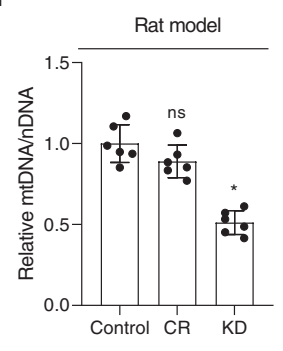

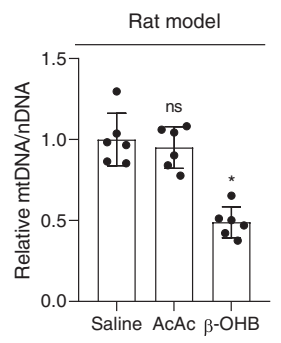

f

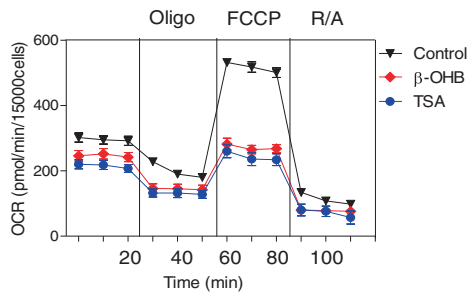

h

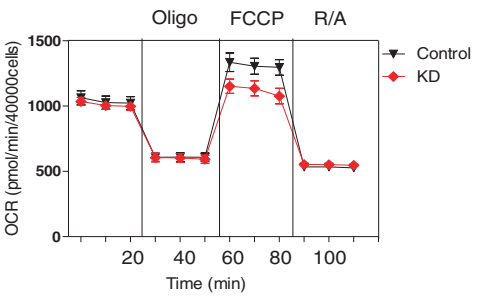

j

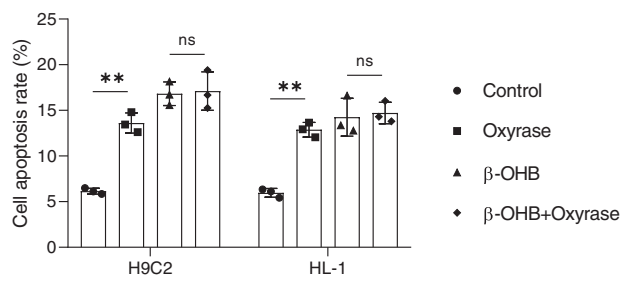

g

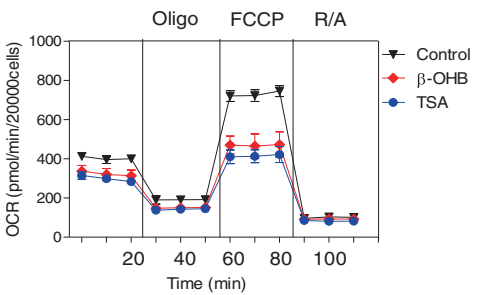

i

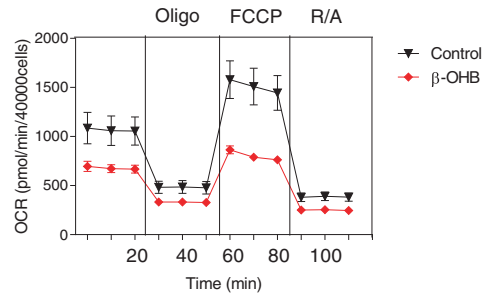

k

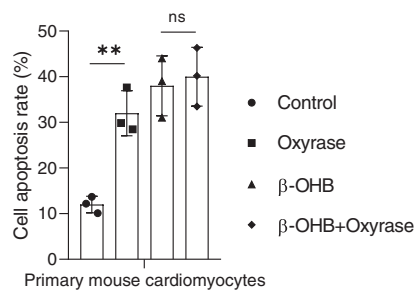

Fig. 3 Increased $\beta-\mathrm{OHB}$ decreased mitochondrion levels. $\mathbf{a}$, b Mitochondrial mass, as determined by MitoTracker Green staining (a) and mitochondrial DNA quantification (b) in cells with different concentrations of $\beta-\mathrm{OHB} ; n=3$. c Mitochondrial mass determined by mitochondrial DNA quantification in mouse primary cardiomyocytes; $n=3$. d, e Mitochondrial mass determined by mitochondrial DNA quantification in atrial tissues from rats with different treatments; $n=6 /$ group. $\mathbf{f}$, g Oxygen consumption rates (OCRs) in H9C2 (f) and HL- 1 (g) cells treated with $\beta-\mathrm{OHB}$ or trichostatin $\mathrm{A}(\mathrm{TSA})$. $\mathbf{h}, \mathbf{i}$ OCRs in primary cardiomyocytes isolated from the atrial tissues of rats with various treatments. j, k Apoptosis in cell lines and primary mouse cardiomyocytes treated with $\beta$-OHB and oxyrase; $n=3$. Data shown are means \pm standard errors. ${ }^{\text {ns }} p>0.05,{ }^{*} p<0.05,{ }^{* *} p<0.01,{ }^{* *} p<0.001$ versus the corresponding control group according to Student's $t$ tests 


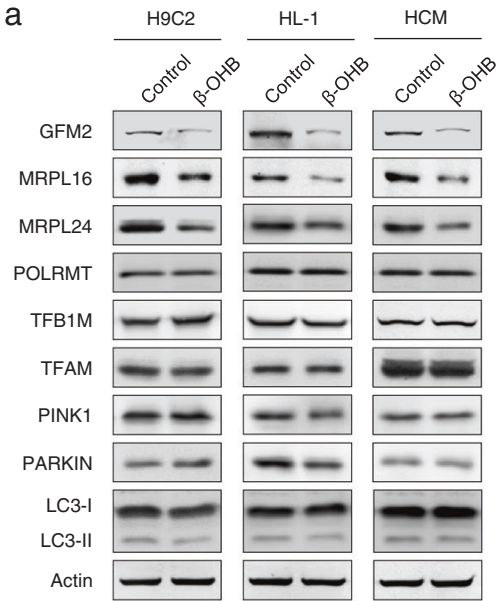

b

b

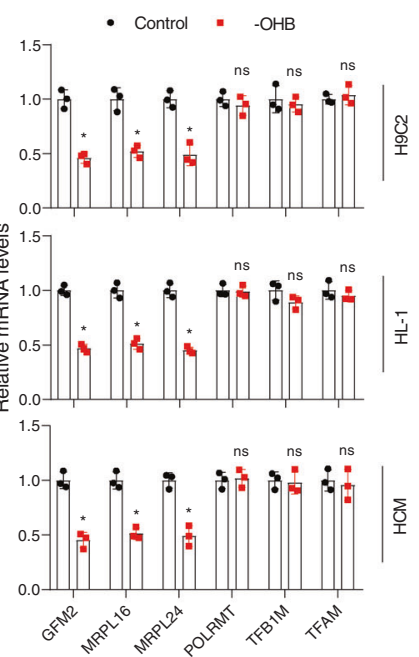

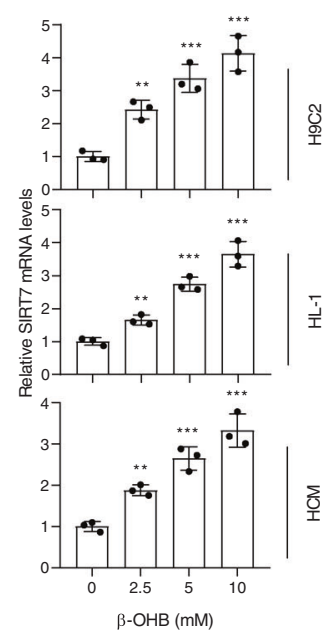

d

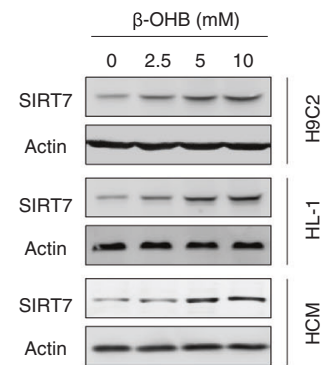

e

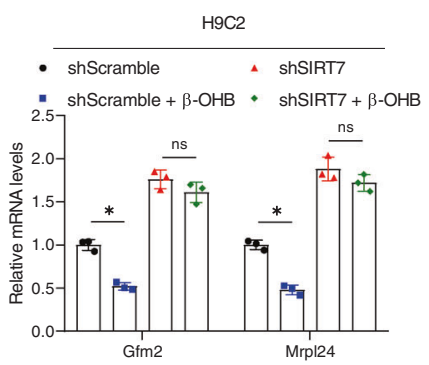

f

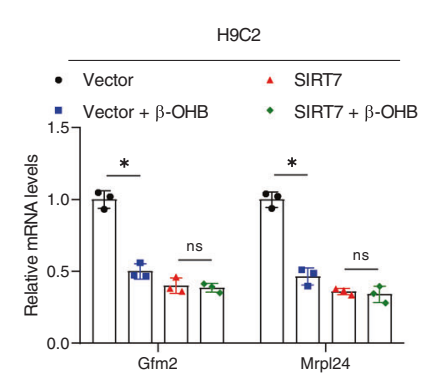

g

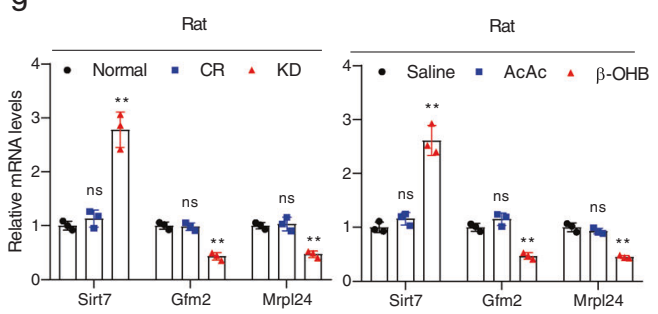

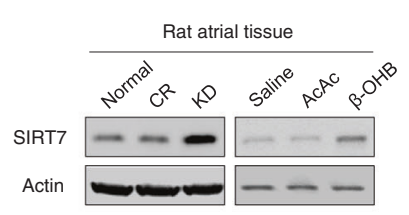

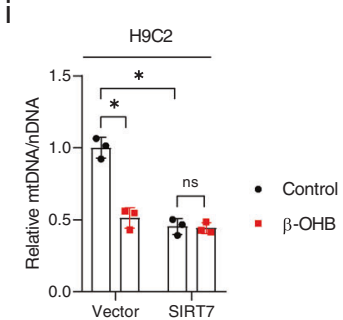

j

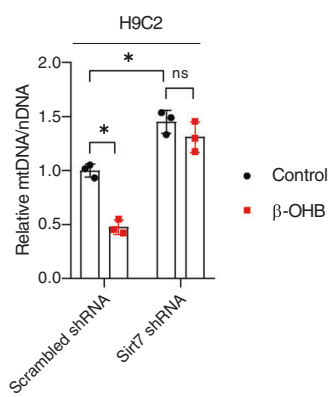

$\mathrm{k}$

Rat atrial tissue

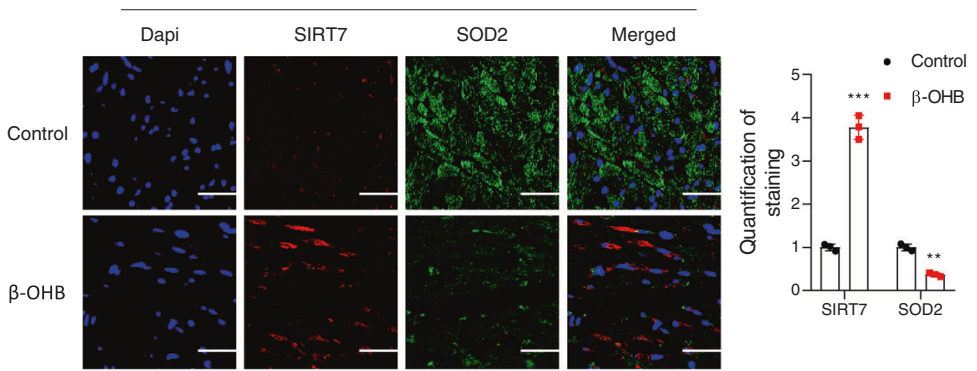

overexpression of SIRT7 resulted in decreased Gfm2 and Mrp/24 transcription, and saturated the effects of $\beta-\mathrm{OHB}$ treatment (Fig. 4f). Moreover, rats with high levels of $\beta-\mathrm{OHB}$, including $\mathrm{KD}$-fed and $\beta$-OHB-injected rats, exhibited increased cardiac SIRT7 mRNA and protein expression (Fig. 4g, h), as well as decreased Gfm2 and Mrp/24 mRNA levels (Fig. 4g). Furthermore, overexpression of SIRT7 decreased mitochondrion levels, whereas knockdown of SIRT7 slightly increased mitochondrion levels in cultured cells (Fig. 4i, j). Either overexpressing or knocking down SIRT7 in cells attenuated the inhibitory effects of $\beta$-OHB treatment (Fig. $4 \mathrm{i}, \mathrm{j}$ ). Double immunofluorescence staining of atrial tissues from $\beta$-OHB-injected or normal rats revealed that $\beta-\mathrm{OHB}$ 
Fig. $4 \quad \beta$-OHB impaired mitochondrial biogenesis by activating SIRT7. a Western blotting analysis of proteins involved in mitochondrial biogenesis and autophagy in different cell lines treated with or without $\beta$-OHB. $\mathbf{b}$ Analysis of mRNA expression of genes involved in the mitochondrial biogenesis pathway in different cell lines treated with or without $\beta-\mathrm{OHB} ; n=3$. c Sirt7 mRNA levels in different cell lines treated with different concentrations of $\beta$-OHB. d SIRT7 protein levels in different cell lines treated with different concentrations of $\beta$-OHB. e Gfm 2 and Mrpl24 mRNA levels in SIRT7-knockdown H9C2 and control cells treated with or without $\beta$-OHB; $n=3$. f Gfm2 and Mrp/24 mRNA levels in SIRT7overexpressing $\mathrm{H} 9 \mathrm{C} 2$ cells and control cells treated with or without $\beta-\mathrm{OHB} ; n=3$. g Sirt7, Gfm2, and Mrpl24 mRNA levels in atrial tissues from rats with different treatments. $\mathbf{h}$ SIRT7 protein levels in rat atrial tissues according to different treatments. $\mathbf{i}$, $\mathbf{j}$ Mitochondrial mass determined by mitochondrial DNA quantification in $\mathrm{H} 9 \mathrm{C} 2$ cells with different treatments; $n=3$. $\mathbf{k}$ Double immunofluorescence staining of SIRT7 and SOD2 in rat atrial tissues. Right panel shows the quantitative results of three replicates. Data shown are means \pm standard errors. ${ }^{\mathrm{ns}} p>0.05,{ }^{*} p<0.05,{ }^{* *} p<0.01,{ }^{* * *} p<0.001$ versus the control group according to Student's $t$ tests. Some full-length blots are presented in the Supplementary Fig. 13

promoted SIRT7 expression and reduced mitochondrial numbers, as indicated by measuring the level of superoxide dismutase 2 (SOD2); this further indicated that $\beta$-OHB impaired mitochondrial biogenesis by activating SIRT7 (Fig. 4k). Furthermore, although high $\beta-O H B$ induced SIRT4 overexpression, forced SIRT4 overexpression had no effect on Gfm2 or Mrp/24 expression (Supplementary Fig. 8b). These results collectively confirmed that $\beta-O H B$ inhibited mitochondrial biogenesis by promoting SIRT7 overexpression.

\section{$\beta$-OHB inhibited histone deacetylase 2 and activated Sirt7} transcription

We next investigated how $\beta-\mathrm{OHB}$ regulated the transcription of Sirt7. We hypothesized that histone acetylation may play a role, as $\beta-\mathrm{OHB}$ is an established inhibitor of histone deacetylases (HDACs), include HDAC1, HDAC3, HDAC4, and HDAC6. ${ }^{12}$ Knockdown of HDACs individually in $\beta-\mathrm{OHB}$-treated $\mathrm{H} 9 \mathrm{C} 2$ cells revealed that only Hdac2 knockdown increased Sirt7 expression and abrogated the ability of $\beta-\mathrm{OHB}$ to activate Sirt7 transcription (Fig. 5a and Supplementary Fig. 9). In turn, overexpression of HDAC2, but not other HDACs, inhibited Sirt7 transcription in $\mathrm{H} 9 \mathrm{C} 2$ cells (Fig. 5b), indicating that HDAC2 was the HDAC that regulated Sirt7 transcription. Accordingly, using chromatin immunoprecipitation (ChIP), we found that HDAC2 bound to the core promoter region of Sirt7 and localized to the same region as acetylated $\mathrm{H} 3 \mathrm{~K} 9$ (Fig. 5c). Moreover, the acetylation levels of H3K9 and H3K14 in the promoter of Sirt7 were increased in $\beta$-OHB-treated cultured $\mathrm{H} 9 \mathrm{C} 2$ cells (Fig. $5 \mathrm{~d}$ ). In addition, we observed increased acetylation levels of H3K9 and H3K14 in the promoter of Sirt7 in the heart tissues of KD-fed and $\beta$-OHB-injected rats, when compared with those in the corresponding control rats (Fig. 5e). These results suggested that Sirt7 transcriptional activation was related to alterations in acetylation, which were regulated by the $\beta-\mathrm{OHB} /$ HDAC2 pathway.

Although $\beta-\mathrm{OHB}$ was previously shown to be negatively correlated with cellular HDAC2 activity, ${ }^{41}$ direct evidence has not yet been obtained to validate whether $\beta$-OHB inhibits HDAC2 deacetylase activity. Therefore, we conducted an in vitro deacetylation assay and found that $\beta-\mathrm{OHB}$ had significant inhibitory effects on HDAC2, with an $\mathrm{IC}_{50}$ of $2.4 \mathrm{mM}$ in vitro (Fig. 5f), suggesting that KD-induced elevation of $\beta-O H B$ in the heart decreased HDAC2 activity by more than half. In cultured cells, we confirmed that $\beta-\mathrm{OHB}$ treatment resulted in increased levels of total histone acetylation (Fig. $5 \mathrm{~g}$ ), particularly of $\mathrm{H} 3 \mathrm{~K} 9$ and H3K14, downstream targets of HDAC2 (Fig. 5h). Trichostatin $A$, a general HDAC inhibitor, caused phenotypes similar to those of $\beta-O H B$, including activation of Sirt7 transcription (Supplementary Fig. 10a), downregulation of Gfm2 and Mrpl24 expression (Supplementary Fig. 10a), inhibition of mitochondrial biogenesis (Supplementary Fig. 10b), inhibition of mitochondrial respiration (Fig. 3f, g), and promotion of apoptosis in cardiomyocytes (Fig. $2 a-c)$. In addition, we confirmed that the expression of voltage-dependent T-type calcium channel subunit alpha-1H (CACNA1H) and voltage-dependent T-type calcium channel subunit alpha-2 delta-2 (CACNA2D2), which are known to be inhibited by HDAC2, ${ }^{42}$ were increased in $\beta$-OHB-treated cells (Fig. 5i) and heart tissues from KD-fed rats (Fig. 5j). Taken together, these results indicated that $\beta-\mathrm{OHB}$ activated Sirt7 transcription by inhibiting HDAC2.

Increased $\beta-\mathrm{OHB}$ and its consequences were observed in patients with $\mathrm{AF}$

In order to confirm the causal role of $\beta-\mathrm{OHB}$ in cardiac fibrosis, we examined cardiac fibrosis, $\beta-\mathrm{OHB}$ levels, and changes in $\beta$-OHB-associated markers in patients with AF. First, we found that $\beta$-OHB levels in cardiac tissues were 3.3 -fold higher in patients with AF than in those with sinus rhythm (SR; Fig. 6a). Second, CACNA1H and CACNA2D2 expression was higher in cardiac tissues from patients with AF, indicating that HDAC2 was inhibited in the cardiac tissues of these patients (Fig. 6b and Supplementary Fig. 11a). Third, increased levels of SIRT7 were observed in cardiac tissues from patients with $\mathrm{AF}$ compared with those in patients with SR, according to both western blotting (Fig. $6 \mathrm{~b}$ and Supplementary Fig. 11a) and IHC (Fig. $6 \mathrm{c}$ and Supplementary Fig. 11b). Fourth, markers of fibrosis, including type I collagen, type III collagen, and a-SMA, were higher in patients with AF (Fig. $6 \mathrm{~b}$ and Supplementary Fig. 11a). Lastly, the number of mitochondria was significantly lower in cardiac tissues from patients with $\mathrm{AF}$ than in those from patients with $\mathrm{SR}$, as indicated by the ratio of $\mathrm{mtDNA}$ to nucleic DNA (Fig. 6d). These findings, together with the observation that the cardiac $\beta-\mathrm{OHB}$ concentration was negatively correlated with the number of mitochondria (Fig. 6e), confirmed that elevations in $\beta-\mathrm{OHB}$ were associated with cardiac fibrosis and an increased risk of $A F$.

\section{DISCUSSION}

Some studies have indicated that $\beta-O H B$ exhibits beneficial effects in the cardiac system under pathological conditions and can, for example, be used as an alternative fuel in the human failing heart. ${ }^{43}$ Moreover, this compound improves cardiac cell excitation-contraction coupling during hypoxia ${ }^{44}$ and prevents myocardial damage after coronary occlusion in animal model. ${ }^{45}$ In this study, we provided evidence and mechanistic explanations from cultured cells, animal models, and clinical samples to show that long-term KD-induced $\beta-\mathrm{OHB}$ accumulation was detrimental to heart health by promoting cardiac fibrosis (Fig. 6f).

Several facts support our findings. First, a KD forces cells in the body to rely primarily on fatty acid $\beta$-oxidation rather than glycolysis for energy production, inevitably leading to increased ketone body production, primarily in the liver; this change elevates circulating levels of ketone bodies and exposes cardiomyocytes in the heart to high levels of ketone bodies. However, although theoretically all cells in the body are exposed to elevated levels of ketone bodies, cardiomyocytes are among those most vulnerable to high ketone body exposure, as high levels of ketone bodies reduce the mitochondrial content significantly, as demonstrated in our study. The mitochondrial content in cardiomyocytes reaches up to $30 \%$ of the total cell 
a

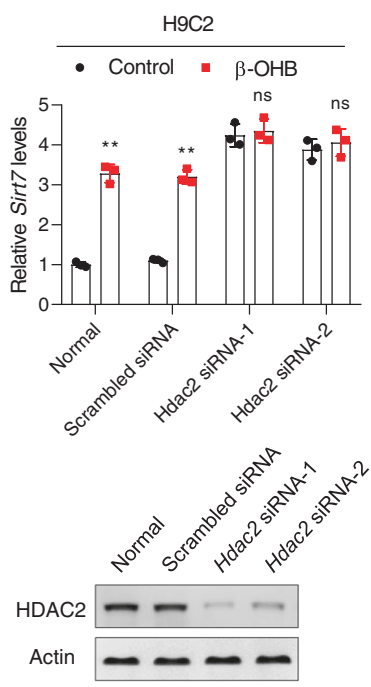

d

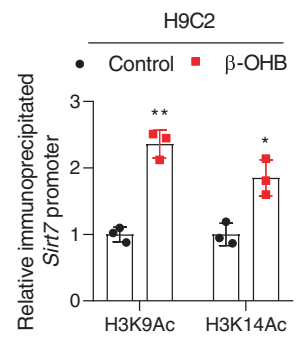

g

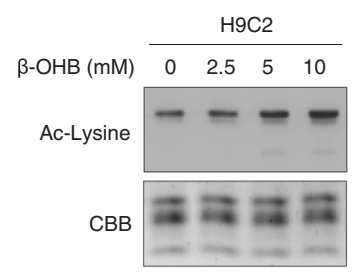

i

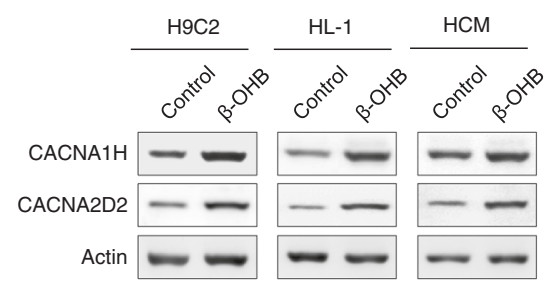

volume, much higher than that in the cells of other organs, because the heart requires high levels of energy production from the $\beta$-oxidation of fatty acids. ${ }^{46}$ Therefore, although the regulatory effects of $\beta-\mathrm{OHB}$ to mitochondrial content may be present in many organs because of HDAC2 and SIRT7 expression
C

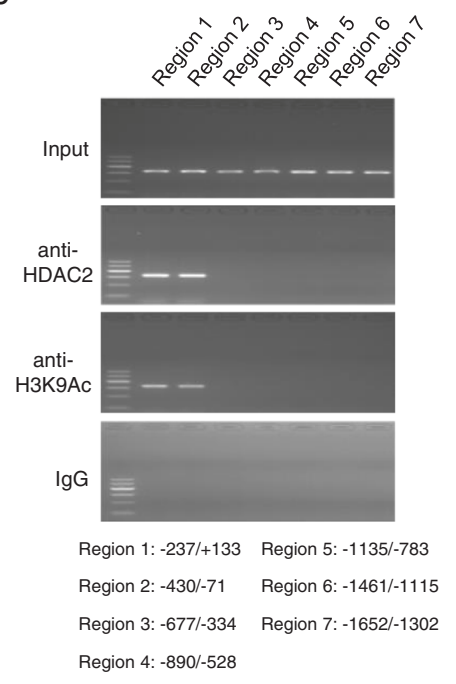

f

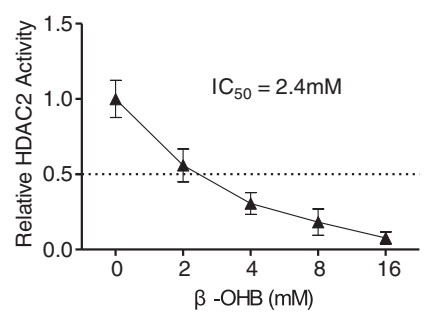

h

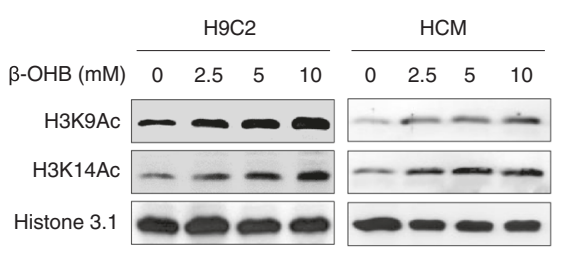

j

\begin{tabular}{|c|c|c|c|c|c|c|c|c|c|c|c|c|}
\hline & & & & & Rat & t atria & I tissu & & & & & \\
\hline & & & Norma & al diet & & & & & etoge & nic die & & \\
\hline & 1 & 2 & 3 & 4 & 5 & 6 & 1 & 2 & 3 & 4 & 5 & 6 \\
\hline CACNA1H & - & - & - & - & - & - & $=$ & - & - & - & - & $=$ \\
\hline CACNA2D2 & - & - & - & - & - & - & - & - & - & $\longrightarrow$ & - & - \\
\hline Actin & $\rightarrow$ & 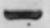 & $=$ & - & - & - & - & $=$ & 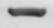 & - & - & - \\
\hline
\end{tabular}

(Supplementary Fig. 12), long-term $\beta$-OHB exposure selectively induced apoptosis of cardiomyocytes.

In addition, HDAC2, which was found to be inactivated by $\beta-O H B$ in our study, is essential for promoting heart development and maintaining heart function, ${ }^{47-49}$ and downregulation 
Fig. $5 \beta$-OHB activated Sirt7 transcription by inhibiting HDAC2. a Sirt7 mRNA levels in Hdac2-knockdown or control H9C2 cells treated with or without $\beta-\mathrm{OHB} ; n=3$. The lower panel shows the knockdown efficiency. b Sirt7 mRNA levels in H9C2 cells overexpressing different histone deacetylases (HDACs); $n=3$. The lower panel shows the knockdown efficiency. c ChIP followed by PCR showing HDAC2 and H3K9Ac occupancy at the Sirt7 promoter. d ChIP followed by qRT-PCR showing the binding affinities of H3K9Ac and H3K14Ac to the Sirt7 promoter in cells treated with or without $\beta-\mathrm{OHB} ; n=3$. e ChIP followed by qRT-PCR showing the binding affinities of H3K9Ac and H3K14Ac to the Sirt7

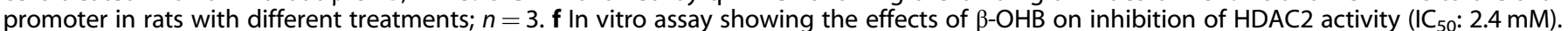
g Total histone acetylation levels in $\mathrm{H} 9 \mathrm{C} 2$ cells treated with different concentrations of $\beta$-OHB. h H3K9Ac and H3K14Ac levels in H9C2 and HCM cells treated with different concentrations of $\beta-O H B$. $\mathbf{i}$ CACNA1H and CACNA2D2 levels in different cells treated with or without $\beta$-OHB. j CACNA1H and CACNA2D2 levels in atrial tissues from rats fed a normal diet or KD. Data shown are means \pm standard errors. ${ }^{\text {ns }} p>0.05,{ }^{*} p<$ $0.05,{ }^{* *} p<0.01,{ }^{* * *} p<0.001$ versus the corresponding control group according to Student's $t$ tests. Some full-length blots are presented in the Supplementary Fig. 13

of HDAC2 increases apoptosis in cardiomyocytes. ${ }^{42}$ Our in vitro assay showed that $\beta-\mathrm{OHB}$ inhibited HDAC2, with an $\mathrm{IC}_{50}$ of $2.4 \mathrm{mM}$, which is lower than the $\beta-\mathrm{OHB}$ concentrations observed in the human AF heart and in KD-fed and deepfasted rats (i.e., $\sim 3-4 \mathrm{mM}$ ). These results further supported that consumption of a KD induced the accumulation of $\beta-\mathrm{OHB}$ to pathological levels.

Although the $\beta$-OHB/HDAC2/SIRT7-mitochondrial biogenesiscardiac fibrosis pathway was found in the current study, several questions remained unsolved. First, systematic metabolic remodeling induced by $\mathrm{KD}$ is more extensive than $\beta-\mathrm{OHB}$ intraperitoneal injection. For example, KD induced decreased levels of glucose and increased levels of free fatty acid in blood, and increased gluconeogenesis in liver and kidney. In contrast, $\beta-\mathrm{OHB}$ intraperitoneal injection did not cause such glucose/fatty acid metabolic reprogram in rat. Although our studies indicated that increased $\beta-O H B$ was able to cause cardiac fibrosis in rat, whether the dysregulation of other types of metabolites induced by longterm KD contributes to cardiac fibrosis remains unknown. Secondly, in the current study, we did not examine whether long-term $\mathrm{KD}$ or $\beta-\mathrm{OHB}$ injection caused other organ abnormalities. So the safety of long-term effects of KD and $\beta-O H B$ injection still required further studies. Third, besides SIRT7, HDAC2 has many downstream targets. In the current study, we aimed to confirm that the $\beta-O H B / H D A C 2 / S I R T 7$ pathway was important to mitochondrial biogenesis. Therefore, we did not measure other cardiac fetal genes that may be regulated by HDAC2. Further studies are required to reveal regulatory effects of HDAC2 on mitochondrial genes. Last, we validated that SIRT7 inhibited mitochondrial biogenesis in cardiomyocytes, consistent with previous findings showing that increased expression or activity of SIRT7 inhibited mitochondrial biogenesis in hematopoietic stem cells and human embryonic kidney cells. ${ }^{40,50}$ However, SIRT7 deficiency in fibroblasts has been shown to induce mitochondrial dysfunction. ${ }^{51}$ Thus, these contradictory observations indicated that SIRT7 may exert different regulatory effects on mitochondrial function in different types of cells.

The findings of our current study defined the mechanism underlying the negative health effects of KDs, which are adopted worldwide for therapeutic purposes and have been increasingly preferred by healthy individuals in order to prevent obesity. Our results strongly suggest that unless the adverse effects of a KD on the cardiac system can be effectively avoided, healthy individuals should reconsider the use of a KD for weight loss.

\section{MATERIALS AND METHODS}

Animal models

Adult male Sprague-Dawley rats weighing 180-220 g were purchased from the Experimental Animal Center of Anhui Medical University. Detailed descriptions of KD-feeding model, ketone body intraperitoneal injection model, and frequent deep fasting model, are given in the Supplementary Methods online.
Clinical samples

For western blotting and metabolite quantification, 30 atrial appendages from patients with rheumatic AF (AF group; $A F$ lasting for $>6$ months), and 30 atrial appendages from patients with SR (SR group) were collected during heart valve replacement surgery or cardiac catheterization conducted between June 2016 and May 2017 at the Department of Cardiothoracic Surgery, The Second Hospital of Anhui Medical University. All patients with AF and SR were matched for sex and age. In the surgery, atrial tissue samples were removed after valve replacement was complete and the incision area was repaired. In cardiac catheterization, left atrial appendage tissue was removed from the heart with a stapler. The removed atrial tissue samples were collected and stored at $-80^{\circ} \mathrm{C}$.

\section{Statistics}

Statistical analysis was performed using Prism 6.0 software (GraphPad Software, Inc.), Excel (Microsoft Corp.), and R version 2.17. Results were expressed as means \pm standard deviations or standard errors of the means. One-way analysis of variance followed by pairwise comparisons using least significant difference test was performed to test between-groups differences, and two-tailed Student's $t$ test was performed for the two-group analysis. Differences were considered statistically significant if the $p$ value was $<0.05$.

\section{Study approval}

All animal experiments and procedures conformed to the guidelines of Directive 2010/63/EU of the European Parliament on the Protection of Animals Used for Scientific Purposes or the NIH guidelines. All animal experimental procedures were approved by the Animal Care and Use Committee of Anhui Medical University. All human samples experimental procedures were reviewed and approved by the ethics committee of Anhui Medical University. Written informed consent was obtained from all eligible patients, and this study conformed to the guidelines outlined in the Declaration of Helsinki.

\section{Experimental setup}

Detailed descriptions of the experimental setup and chemicals, including echocardiography, histopathology, IHC, cell culture and treatments, cardiac fibroblast and cardiomyocyte isolation and culture, antibodies, plasmid construction and transfection, RNA interference, immunoprecipitation and immunoblotting, ChIP assays, quantitative real-time reverse transcription (qRT)$P C R$, in vitro HDAC activity assay, histone extraction, gas chromatography/mass spectrometry, measurements of the concentrations of ketone bodies, apoptosis assay, TUNEL assay, measurements of mitochondrial mass and mtDNA/nDNA, mtDNA damage quantification, measurements of oxygen consumption, and Immunofluorescence, are given in the Supplementary Methods online. 

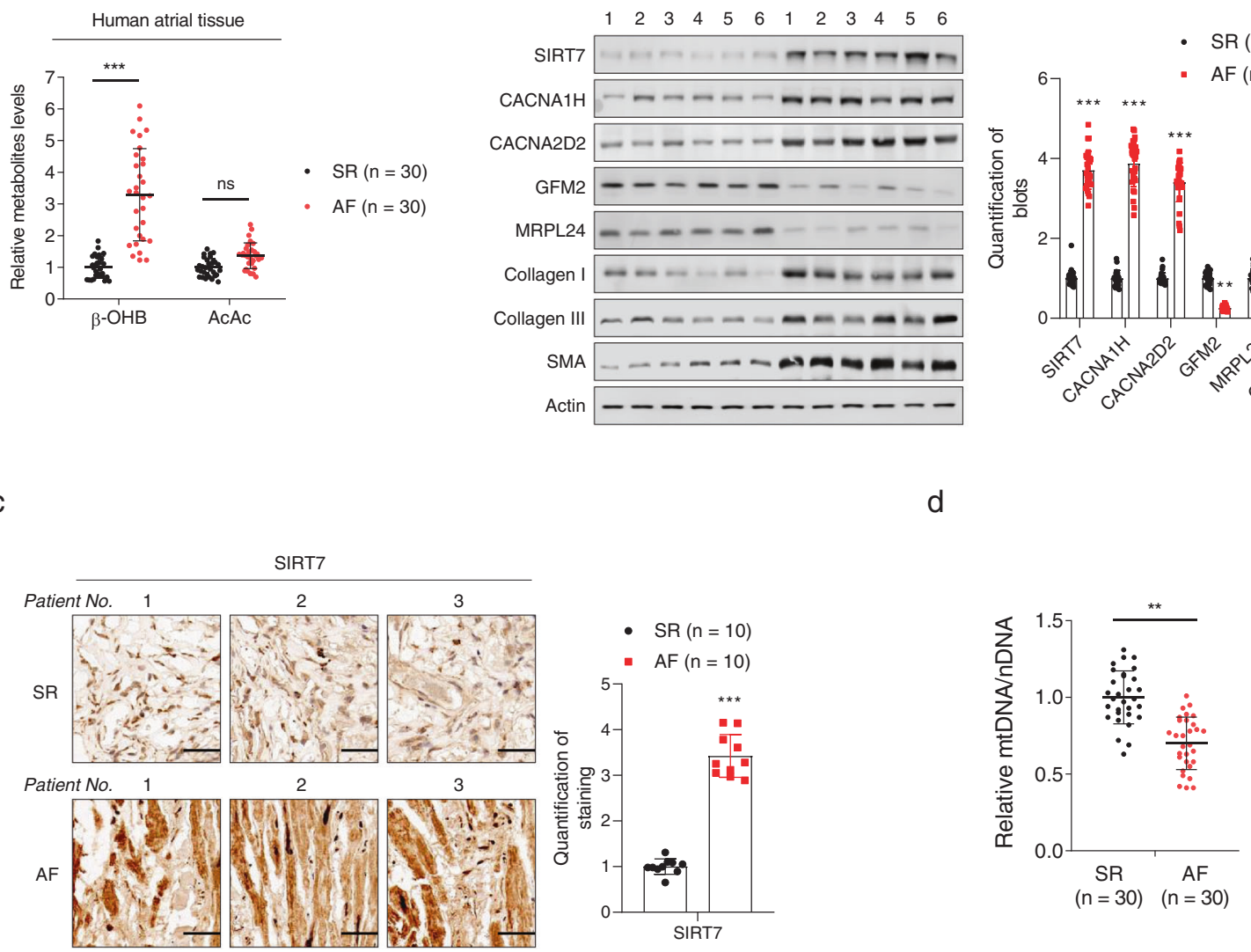

e

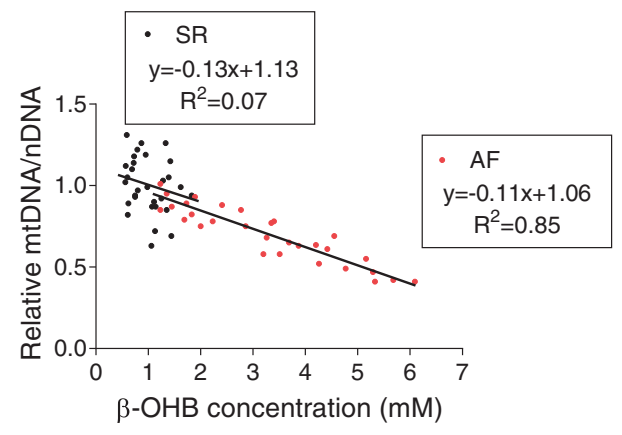

b

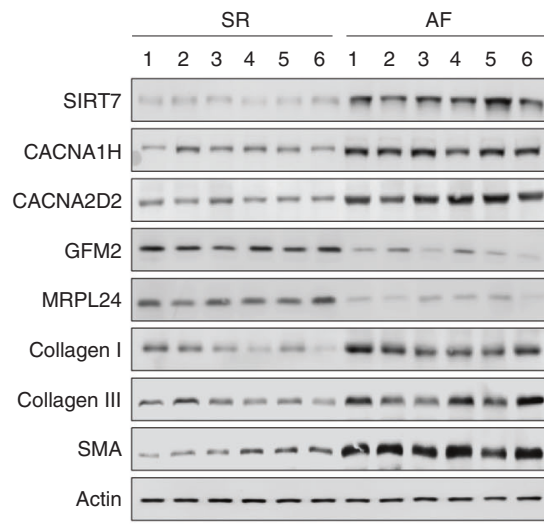

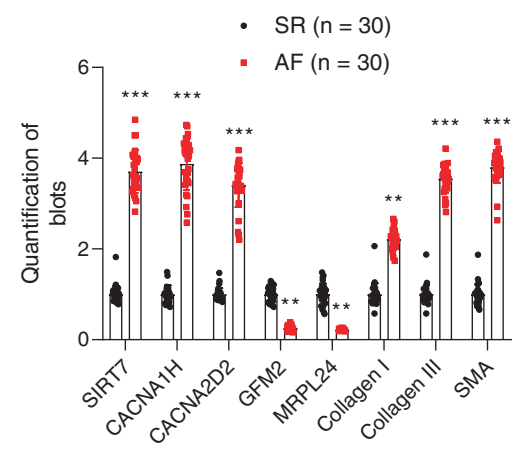




\section{DATA AVAILABILITY}

The data sets used for the current study are available from the corresponding author upon reasonable request.

\section{ACKNOWLEDGEMENTS}

This work was supported by the Grants from the State Key Development Programs of Basic Research of China (Nos. 2019YFA0801900, 2018YFC100242, 2018YFA080030, 2018YFA0801300, and 2018YFC1004700), the National Natural Science Foundation of China (Nos. 31871432, 81771627, 31521003, 31821002, 91753207, 81722021, $81471454,31671483,31671453$, and 31425008), and grant from Key Laboratory of Reproduction Regulation of NPFPC.

\section{AUTHOR CONTRIBUTIONS}

J.-Y.Z. conceived the study, and designed and supervised the experiments; S.X., H.T., Y.L., W.X., and S.-M.Z. performed the experiments and analyzed the data; H.T., W.C., and J.C. generated the animal models and collected the clinic samples. J.-Y.Z. wrote the manuscript. All authors read and discussed the manuscript.

\section{ADDITIONAL INFORMATION}

The online version of this article (https://doi.org/10.1038/s41392-020-00411-4) contains supplementary material, which is available to authorized users.

Competing interests: The authors declare no competing interests.

\section{REFERENCES}

1. Neal, E. G. et al. The ketogenic diet for the treatment of childhood epilepsy: a randomised controlled trial. Lancet Neurol. 7, 500-506 (2008).

2. Nielsen, J. V. \& Joensson, E. A. Low-carbohydrate diet in type 2 diabetes: stable improvement of bodyweight and glycemic control during 44 months follow-up. Nutr. Metab. 5, 14 (2008).

3. Allen, B. G. et al. Ketogenic diets enhance oxidative stress and radio-chemotherapy responses in lung cancer xenografts. Clin. Cancer Res. 19, 3905-3913 (2013).

4. Otto, C. et al. Growth of human gastric cancer cells in nude mice is delayed by a ketogenic diet supplemented with omega-3 fatty acids and medium-chain triglycerides. BMC Cancer 8, 122 (2008).

5. Stafstrom, C. E. \& Rho, J. M. The ketogenic diet as a treatment paradigm for diverse neurological disorders. Front. Pharmacol. 3, 59 (2012).

6. Bueno, N. B., de Melo, I. S., de Oliveira, S. L. \& Da, R. A. T. Very-low-carbohydrate ketogenic diet v. low-fat diet for long-term weight loss: a meta-analysis of randomised controlled trials. Br. J. Nutr. 110, 1178-1187 (2013).

7. Cahill, G. J. et al. Hormone-fuel interrelationships during fasting. J. Clin. Investig. 45, 1751-1769 (1966).

8. Robinson, A. M. \& Williamson, D. H. Physiological roles of ketone bodies as substrates and signals in mammalian tissues. Physiol. Rev. 60, 143-187 (1980).

9. Cahill, G. J. Fuel metabolism in starvation. Annu. Rev. Nutr. 26, 1-22 (2006)

10. Johnson, R. H., Walton, J. L., Krebs, H. A. \& Williamson, D. H. Post-exercise ketosis. Lancet 2, 1383-1385 (1969).

11. Cox, P. J. et al. Nutritional ketosis alters fuel preference and thereby endurance performance in athletes. Cell Metab. 24, 256-268 (2016).

12. Shimazu, T. et al. Suppression of oxidative stress by beta-hydroxybutyrate, an endogenous histone deacetylase inhibitor. Science 339, 211-214 (2013).

13. Han, Y. M. et al. beta-Hydroxybutyrate prevents vascular senescence through hnRNP A1-mediated upregulation of Oct4. Mol. Cell. 71, 1064-1078 (2018).

14. Youm, Y. H. et al. The ketone metabolite beta-hydroxybutyrate blocks NLRP3 inflammasome-mediated inflammatory disease. Nat. Med. 21, 263-269 (2015).

15. Mayr, M. et al. Combined metabolomic and proteomic analysis of human atrial fibrillation. J. Am. Coll. Cardiol. 51, 585-594 (2008).

16. Obokata, M. et al. Association between circulating ketone bodies and worse outcomes in hemodialysis patients. J. Am. Heart Assoc. 6 (2017).

17. Lau, D. H., Nattel, S., Kalman, J. M. \& Sanders, P. Modifiable risk factors and atrial fibrillation. Circulation 136, 583-596 (2017).

18. Wilson, P. W. et al. Prediction of coronary heart disease using risk factor categories. Circulation 97, 1837-1847 (1998).

19. Bergqvist, A. G., Chee, C. M., Lutchka, L., Rychik, J. \& Stallings, V. A. Selenium deficiency associated with cardiomyopathy: a complication of the ketogenic diet. Epilepsia 44, 618-620 (2003).
20. Best, T. H., Franz, D. N., Gilbert, D. L., Nelson, D. P. \& Epstein, M. R. Cardiac complications in pediatric patients on the ketogenic diet. Neurology 54, 2328-2330 (2000).

21. Kang, H. C., Chung, D. E., Kim, D. W. \& Kim, H. D. Early- and late-onset complications of the ketogenic diet for intractable epilepsy. Epilepsia 45, 1116-1123 (2004).

22. Seidelmann, S. B. et al. Dietary carbohydrate intake and mortality: a prospective cohort study and meta-analysis. Lancet Public Health 3, e419-e428 (2018).

23. Clarke, R. et al. Life expectancy in relation to cardiovascular risk factors: 38 year follow-up of 19,000 men in the Whitehall study. BMJ 339, b3513 (2009).

24. O'Hanlon, R. et al. Prognostic significance of myocardial fibrosis in hypertrophic cardiomyopathy. J. Am. Coll. Cardiol. 56, 867-874 (2010).

25. Andersen, S., Nielsen-Kudsk, J. E., Vonk, N. A. \& de Man, F. S. Right ventricular fibrosis. Circulation 139, 269-285 (2019).

26. Galati, G. et al. Histological and histometric characterization of myocardial fibrosis in end-stage hypertrophic cardiomyopathy: a clinical-pathological study of 30 explanted hearts. Circ. Heart Fail. 9 (2016).

27. Nattel, S. Molecular and cellular mechanisms of atrial fibrosis in atrial fibrillation. JACC Clin. Electrophysiol. 3, 425-435 (2017).

28. Jacoby, J. J. et al. Cardiomyocyte-restricted knockout of STAT3 results in higher sensitivity to inflammation, cardiac fibrosis, and heart failure with advanced age. Proc. Natl Acad. Sci. USA 100, 12929-12934 (2003).

29. Singh, V. P., Le, B., Khode, R., Baker, K. M. \& Kumar, R. Intracellular angiotensin II production in diabetic rats is correlated with cardiomyocyte apoptosis, oxidative stress, and cardiac fibrosis. Diabetes 57, 3297-3306 (2008).

30. Newman, J. C. \& Verdin, E. Ketone bodies as signaling metabolites. Trends Endocrinol. Metab. 25, 42-52 (2014).

31. Tanaka, M. et al. Hypoxia induces apoptosis with enhanced expression of Fas antigen messenger RNA in cultured neonatal rat cardiomyocytes. Circ. Res. 75, 426-433 (1994).

32. Long, X. et al. p53 and the hypoxia-induced apoptosis of cultured neonatal rat cardiac myocytes. J. Clin. Investig. 99, 2635-2643 (1997).

33. Bialik, S. et al. The mitochondrial apoptotic pathway is activated by serum and glucose deprivation in cardiac myocytes. Circ. Res. 85, 403-414 (1999).

34. Olivetti, G. et al. Apoptosis in the failing human heart. N. Engl. J. Med. 336, 1131-1141 (1997).

35. Saraste, A. et al. Apoptosis in human acute myocardial infarction. Circulation 95 320-323 (1997).

36. Zhao, J. et al. The different response of cardiomyocytes and cardiac fibroblasts to mitochondria inhibition and the underlying role of STAT3. Basic Res. Cardiol. 114, 12 (2019).

37. Goldenthal, M. J. Mitochondrial involvement in myocyte death and heart failure. Heart Fail. Rev. 21, 137-155 (2016).

38. Nguyen, B. Y. et al. Mitochondrial function in the heart: the insight into mechanisms and therapeutic potentials. Br. J. Pharmacol. 176, 4302-4318 (2019).

39. Scarpulla, R. C., Vega, R. B. \& Kelly, D. P. Transcriptional integration of mitochondrial biogenesis. Trends Endocrinol. Metab. 23, 459-466 (2012).

40. Mohrin, M. et al. Stem cell aging. A mitochondrial UPR-mediated metabolic checkpoint regulates hematopoietic stem cell aging. Science 347, 1374-1377 (2015).

41. Sleiman, S. F. et al. Exercise promotes the expression of brain derived neurotrophic factor (BDNF) through the action of the ketone body betahydroxybutyrate. ELife. 5 (2016).

42. Montgomery, R. L. et al. Histone deacetylases 1 and 2 redundantly regulate cardiac morphogenesis, growth, and contractility. Genes Dev. 21, 1790-1802 (2007).

43. Aubert, G. et al. The failing heart relies on ketone bodies as a fuel. Circulation 133 , 698-705 (2016).

44. Klos, M., Morgenstern, S., Hicks, K., Suresh, S. \& Devaney, E. J. The effects of the ketone body beta-hydroxybutyrate on isolated rat ventricular myocyte excitation-contraction coupling. Arch. Biochem. Biophys. 662, 143-150 (2019).

45. Zou, Z., Sasaguri, S., Rajesh, K. G. \& Suzuki, R. dl-3-Hydroxybutyrate administration prevents myocardial damage after coronary occlusion in rat hearts. Am. J. Physiol. Heart Circ. Physiol. 283, H1968-H1974 (2002).

46. Piquereau, J. et al. Mitochondrial dynamics in the adult cardiomyocytes: which roles for a highly specialized cell? Front. Physiol. 4, 102 (2013).

47. Trivedi, C. M. et al. Hdac2 regulates the cardiac hypertrophic response by modulating Gsk3 beta activity. Nat. Med. 13, 324-331 (2007).

48. Zhang, H. et al. HDAC2 is required by the physiological concentration of glucocorticoid to inhibit inflammation in cardiac fibroblasts. Can. J. Physiol. Pharmacol. 95, 1030-1038 (2017)

49. Kee, H. J. et al. Activation of histone deacetylase 2 by inducible heat shock protein 70 in cardiac hypertrophy. Circ. Res. 103, 1259-1269 (2008). 
Ketogenic diets inhibit mitochondrial biogenesis and induce cardiac... Xu et al.

50. Yan, W. W. et al. Arginine methylation of SIRT7 couples glucose sensing with mitochondria biogenesis. EMBO Rep. 19 (2018).

51. Ryu, D. et al. A SIRT7-dependent acetylation switch of GABPbeta1 controls mitochondrial function. Cell Metab. 20, 856-869 (2014)

(c) (i) Open Access This article is licensed under a Creative Commons Attribution 4.0 International License, which permits use, sharing, adaptation, distribution and reproduction in any medium or format, as long as you give appropriate credit to the original author(s) and the source, provide a link to the Creative
Commons license, and indicate if changes were made. The images or other third party material in this article are included in the article's Creative Commons license, unless indicated otherwise in a credit line to the material. If material is not included in the article's Creative Commons license and your intended use is not permitted by statutory regulation or exceeds the permitted use, you will need to obtain permission directly from the copyright holder. To view a copy of this license, visit http://creativecommons. org/licenses/by/4.0/.

(c) The Author(s) 2021 